Pacific Journal of Mathematic 


\title{
WREATH PRODUCTS OF ORDERED PERMUTATION GROUPS
}

\section{W. Charles Holland and Stephen H. McCleary}

\begin{abstract}
In this paper we define the ordered wreath product of a chain of ordered permutation groups and characterize it by certain completeness properties. We then use the construction to prove a nonabelian analogue of Hahn's theorem for totally ordered abelian groups.
\end{abstract}

One of the fundamental tools in the theory of totally ordered groups is Hahn's theorem [4; a detailed discussion may be found in Fuchs [3]], which asserts, roughly, that every abelian totally ordered group can be embedded in a lexico-graphically ordered (unrestricted) direct sum of copies of the ordered group of real numbers. Almost any general question regarding the structure of abelian totally ordered groups can be answered by reference to Hahn's theorem. Although Conrad [2] has used the Schreier theory to describe the structure of certain classes of nonabelian ordered groups, no tool like Hahn's theorem has been available for the general case.

The natural nonabelian analogue of the direct product of two groups (at least for embedding problems) is the wreath product. Lloyd [9] and Reilly [12] have shown that it is possible to lattice-order the wreath product of two lattice-ordered groups. A definition has recently been given [8] for the wreath product of an arbitrary set of groups, and in this paper we shall investigate the consequences of that construction for certain partially ordered groups. Although we shall occasionally refer to theorems proved in [8], familiarity with the material in [8] is not assumed.

One of our results will be an analogue of Hahn's theorem: every totally ordered group can be embedded in a lexicographically ordered wreath product of copies of the ordered group of real numbers. This theorem suffers by comparison with Hahn's theorem in one critical aspect; namely, the wreath product of totally ordered groups is not totally ordered, but only lattice-ordered. Thus, we shall broaden our interest to consider partially ordered groups which are transitive subgroups of the partially ordered group $A(S)$ of all order-preserving permutations of a chain $S$ (ordered by $f \leqq g$ if and only if $s f \leqq s g$ for all $s \in S$ ). This class includes every totally ordered group (permuting itself in the Cayley representation), and a large class of latticeordered groups [6]. We shall define the wreath product of a collection of such ordered permutation groups and show that the wreath product is itself an ordered permutation group. If the factors are lattice- 
ordered, then so is the wreath product.

A transitive group $G$ of order-preserving permutations of a chain $S$ determines a set of " $o$-primitive components" which are themselves ordered permutation groups, and $G$ can be embedded in the ordered wreath product of these components. One corollary of this is the analogue of Hahn's theorem mentioned before. We also characterize ordered wreath products by certain completeness properties.

2. The ordered wreath product of two ordered permutation groups. The set $A(S)$ of all order-preserving permutations (o-permutations) of a chain $S$ becomes a lattice-ordered group when we define $f \leqq g$ if and only if $s f \leqq s g$ for all $s \in S$. The lattice operations are $s(g \vee h)=s g \vee s h$, and dually. If $G$ is a subgroup of $A(S)$ with the inherited partial order, we shall say that $(G, S)$ is an ordered permutation group (o-permutation group).

We want to motivate an investigation of generalized ordered wreath products by a brief discussion of the ordered wreath product of two ordered permutation groups. Most of these definitions and results can be found in [9] and [12]. We shall omit proofs because we shall prove much more general theorems later.

Let $(H, T)$ and $(K, V)$ be $o$-permutation groups. The (unrestricted) wreath product $(H, T) W r(K, V)=(W, R)$ is defined (see, e.g., M. Hall [5]) to be the group of all permutations of $R=T \times V$ of the form $(t, v) \rightarrow\left(t h_{v}, v k\right)$, where $k \in K$ and $h_{v} \in H$. If we order $T \times V$ lexicographically by setting $\left(t_{1}, v_{1}\right) \leqq\left(t_{2}, v_{2}\right)$ if and only if $v_{1}<v_{2}$ or $v_{1}=$ $v_{2}$ and $t_{1} \leqq t_{2}$, then $T \times V$ becomes a chain $\overleftarrow{T \times V}$ and each element of the wreath product becomes an o-permutation of that chain. Thus $W$ is a subgroup of $A(R)$. Moreover, if $H$ and $K$ are both latticeordered (closed under the lattice operation defined above), so is $W$.

Now we describe a certain universal property possessed by the ordered wreath product. If $F$ is any set of permutations of a chain $S$, then a convex $F$-congruence on $S$ is an equivalence relation $Q$ on $S$ such that each $Q$-class is convex $(x \leqq y \leqq z$ and $x Q z$ implies $x Q y)$ and such that if $x Q z$ and $f \in F$, then $(x f) Q(z f)$. Now let $(G, S)$ be a transitive o-permutation group. If $g \in G$ and $s \in S$, then $g \mid s Q$ is an order isomorphism (o-isomorphism) of the chain $s Q$ upon the chain $(s g) Q$, so all $Q$-classes are $o$-isomorphic. Let $T=s Q$ be any fixed $Q$ class and let $G_{T}=\{h \in G \mid T h=T\}$. There is a natural $o$-homomorphism of $G_{T}$ into $A(T)$, whereby each $g \in G_{T}$ corresponds to its restriction to $T$. The image of $G_{T}$ under this homomorphism is denoted by $G \mid T$. Thus if $H=G \mid T,(H, T)$ is an o-permutation group.

The set $V=S / Q$ of all $Q$-classes bears a natural total order in which $x Q<y Q$ if and only if $x<y$ and $x Q \neq y Q$. Moreover, each 
$g \in G$ induces a natural $o$-permutation $\bar{g} \in A(V)$, given by $(x Q) \bar{g}=(x g) Q$. The map $g \rightarrow \bar{g}$ is an $o$-homomorphism of $G$ into $A(V)$. If the image of $G$ in $A(V)$ is denoted by $K=G / Q$, then $(K, V)$ is an $o$-permutation group. Since $G$ is transitive on $S$, so is $H$ transitive on $T$ and $K$ transitive on $V$; and all of the permutation groups $(H, T)$ for various $Q$-classes $T$ are $o$-isomorphic. We shall call $(H, T)$ and $(K, V)$ the local and global $Q$-components, respectively, of $(G, S)$.

There is a natural $o$-isomorphism between the chains $S$ and $\overleftarrow{T \times V}$ which can be realized as follows. Fix some point $O \in S$, let $T=O Q$, and for each $Q$-class $x Q$, choose $g_{x Q} \in G$ such that $O g_{x Q} \in x Q$ (by transitivity of $G$ ). Then map $S$ onto $\overleftarrow{T \times V}$ by $s \rightarrow(t, v)$, where $v=s Q$ and $t=s g_{s Q}^{-1}$. Identifying $S$ with $\overleftarrow{T \times V}$ in this way, each $g \in G$ is an element of the ordered wreath product $(W, R)$. Thus $(G, S)$ is embedded in the ordered wreath product of its local and global components. Under the identification of $S$ and $R=\overleftarrow{T \times V}$, the convex $G$-congruence $Q$ is described by $\left(t_{1}, v_{1}\right) Q\left(t_{2}, v_{2}\right)$ if and only if $v_{1}=v_{2}$. Thus $Q$ is also a convex $W$-congruence. Moreover, the local and global components of $(W, R)$ are just $(H, T)$ and $(K, V)$, respectively. Roughly speaking, the wreath product of the $Q$-components of $G$ is gotten by adding to $G$ as many o-permutations of $S$ as possible without changing the $Q$-components.

In the following sections, we shall develop a theory which is more general than the one discussed here in that instead of a single $G$-congruence $Q$ on $S$, we shall have a more or less arbitrary collection of convex $G$-congruences on $S$, producing components which are neither local nor global, but somewhere in between.

3. Construction of the general ordered wreath product. What we define here is a special case of the general wreath product defined for (nonordered) permutation groups in [8]. Let $\Gamma$ be a chain, and for each $\gamma \in \Gamma$, let $S_{\gamma}$ be a chain and $\left(G_{\gamma}, S_{\gamma}\right)$ a transitive o-permutation group. Let $\bar{R}=\prod_{\gamma \in \Gamma} S_{\gamma}$ be the cartesian product, and choose any point $O \in \bar{R}$. Thinking of the elements of $\bar{R}$ as functions on $\Gamma$, we define the support of $v \in \bar{R}$ to be $\{\gamma \in \Gamma \mid r(\gamma) \neq 0(\gamma)\}$. Now let $R=\{r \in \bar{R} \mid r$ has inversely well ordered support $\}$, so that for $r, s \in R$, $\{\gamma \in \Gamma \mid r(\gamma) \neq s(\gamma)\}$ is inversely well ordered. For $r, s \in R, r \neq s$, we define $r<s$ if and only if $r(\alpha)<s(\alpha)$ in $S_{\alpha}$, where $\alpha$ is the largest element of the set $\{\gamma \in \Gamma \mid r(\gamma) \neq s(\gamma)\}$. This makes $R$ a chain.

For each $\gamma \in \Gamma$, we define an equivalence relation $K_{\gamma}$ on $R$ by setting $r K_{\gamma} s$ if and only if $r(\alpha)=s(\alpha)$ for all $\alpha \geqq \gamma$. Let $K^{\gamma}=\bigcap_{\beta>\gamma} K_{\beta}$ (and $K^{\gamma}=R \times R$ if there is no $\beta>\gamma$ ). Equivalently, $r K^{\gamma} s$ if and only if $r(\alpha)=s(\alpha)$ for all $\alpha>\gamma$. All $K_{r}$-classes and all $K^{\gamma}$-classes are convex. 
Let $P(R)$ denote the set of all (not necessarily order-preserving) permutations of $R$. Let $W^{\prime}=\left\{f \in P(R) \mid K_{\gamma}\right.$ is a (convex) $\left\{f, f^{-1}\right\}$ congruence for each $\gamma \in \Gamma\}$, a subgroup of $P(R)$. Then for every $f \in W^{\prime}$ and $\gamma \in \Gamma, K^{r}$ is also a (convex) $\{f\}$-congruence. Clearly $K^{r}$ contains $K_{r}$ and the chain $\left(O K^{r}\right) / K_{r}$ (ordered in the natural way) is o-isomorphic to the chain $S_{r}$.

Let $g \in W^{\prime}, \gamma \in \Gamma$, and $x \in R . \quad g$ induces a permutation $g_{\gamma, x}$ of $S_{\gamma}$, defined in the following way. If $a \in S_{r}$, there exists $x^{\prime} \in R$ such that $x^{\prime} K^{\gamma} x$ and $x^{\prime}(\gamma)=a$. (We need only alter the $\gamma^{\text {th }}$ component of $x$ to obtain $x^{\prime}$.) Now define $a g_{\gamma, x}=\left(x^{\prime} g\right)(\gamma) . g_{\gamma, x}$ is well defined, for if $x^{\prime \prime} K^{\gamma} x$ and $x^{\prime \prime}(\gamma)=a$, then $x^{\prime \prime} K_{\gamma} x^{\prime}$, whence $\left(x^{\prime \prime} g\right) K_{\gamma}\left(x^{\prime} g\right)$, and in particular, $\left(x^{\prime \prime} g\right)(\gamma)=\left(x^{\prime} g\right)(\gamma)$. Thus we have the rules

(1) $(x g)(\gamma)=(x(\gamma)) g_{\gamma, x}$, and

(2) $g_{\gamma, x}=g_{\gamma, y}$ if $x K^{\gamma} y$.

Of course (2) states that $g_{\gamma, x}$ really depends only on $\gamma$ and the congruence class $x K^{\gamma}$.

It is easy to see [8] that $g_{\gamma, x}$ is a permutation of $S_{\gamma}$ and that $g \in W^{\prime}$ is determined by the matrix $\left\{g_{\gamma, x} \mid \gamma \in \Gamma, x \in R\right\}$ of its components.

THEOREM 1. Let $g \in W^{\prime}$. Then $g$ preserves order if and only if all $g_{r, x}$ 's preserve order.

Proof. First, suppose that $g \in A(R)$. Let $a, b \in S_{r}$ with $a<b$ and let $x^{\prime} K^{\gamma} x K^{\gamma} x^{\prime \prime}$ with $x^{\prime}(\gamma)=a$ and $x^{\prime \prime}(\gamma)=b$. It follows that $x^{\prime}<x^{\prime \prime}$, so that $x^{\prime} g<x^{\prime \prime} g$. But as $\left(x^{\prime} g\right) K^{\gamma}\left(x^{\prime \prime} g\right)$, we must have $\left(x^{\prime} g\right)(\gamma)<\left(x^{\prime \prime} g\right)(\gamma)$, i.e., $a g_{r, x}<b g_{r, x}$. Therefore $g_{r, x} \in A\left(S_{r}\right)$.

Conversely, suppose that $g$ is a permutation in $W^{\prime}$ such that for each $\gamma \in \Gamma, x \in R, g_{\gamma, x} \in A\left(S_{\gamma}\right)$. If $x, y \in R$ with $x<y$, let $\gamma$ be the largest element of $\Gamma$ such that $x(\gamma) \neq y(\gamma)$. Then $x(\gamma)<y(\gamma)$; and $x K^{\gamma} y$, so $(x g) K^{\gamma}(y g)$. Now $(x g)(\gamma)=(x(\gamma)) g_{\gamma, x}<(y(\gamma)) g_{\gamma, x}=(y(\gamma)) g_{\gamma, y}=$ $(y g)(\gamma)$. Hence $x g<y g$.

If $\left\{\left(G_{\gamma}, S_{\gamma}\right)\right\}$ is a collection of o-permutation groups indexed by a chain $\Gamma$, we define the ordered wreath product ${ }^{*} \prod_{\gamma \in \Gamma}\left(G_{r}, S_{\gamma}\right)$ to be ( $W, R$ ), where $R$ is as specified above and $W$ is the set of all permutations $g \in W^{\prime}$ such that $g_{\gamma, x} \in G_{\gamma}$ for all $\gamma \in \Gamma, x \in R$.

The ordered wreath product we have defined here is the same as the general wreath product defined in [8]. In [8] it is shown that the general wreath product is a subgroup of $P(R)$; that it is transitive; and that, to within o-isomorphism, it is independent of which element 0 is chosen from $R^{\prime}$. By Theorem 1 , each $g \in W$ preserves order. Thus we have

THEOREM 2. The ordered wreath product $(W, R)={ }^{*} \Pi_{r \in I}\left(G_{\gamma}, S_{\gamma}\right)$ 
of transitive ordered permutation groups $\left(G_{r}, S_{r}\right), \Gamma$ a chain, is a transitive ordered permutation group.

In case $\Gamma$ is a two element chain, say $0<1$, then $R=\bar{R}=S_{0} \times S_{1}$, and the ordered wreath product we have constructed is the same as the one developed by Lloyd and Reilly that was described in $\S 2$.

4. The o-primitive components of an ordered permutation group. Let $\gamma=\left(Q_{r}, Q^{r}\right)$ be a pair of convex $G$-congruences of a transitive o-permutation group $(G, S)$, with $Q_{r}$ properly contained in $Q^{r}$. Let $O$ be any point in $S$. Let $S_{\gamma}$ be the chain $\left(O Q^{r}\right) / Q_{r}$ and let $G_{O Q^{r}}=$ $\left\{g \in G \mid\left(O Q^{r}\right) g=O Q^{r}\right\}$. Let $G_{\gamma}$ be the image in $A\left(S_{\gamma}\right)$ of $G_{O Q^{r}}$ under the natural $o$-homomorphism $g \rightarrow \bar{g}$, where $\left(x Q_{\gamma}\right) \bar{g}=(x g) Q_{r}$. Then $\left(G_{\gamma}, S_{r}\right)$ is called the $\gamma$-component of $(G, S)$. It is an easy consequence of transitivity that, to within 0 -isomorphism, $\left(G_{i}, S_{\gamma}\right)$ is independent of the choice of $O \in S$.

The following argument from [11] generalizes a theorem from [7].

Theorem 3. If $(G, S)$ is a transitive ordered permutation group, the set of all convex G-congruences is a chain under inclusion.

Proof. Since $G$ is transitive, it suffices to pick any $O \in S$ and to show that for any convex $G$-congruences $C$ and $D$, either $O C \subseteq O D$ or $O D \subseteq O C$. Suppose that neither of these two convex sets is contained in the other. By transitivity, we may pick $g \in G$ such that $O g=d \in O D \backslash O C$. Since $O g$

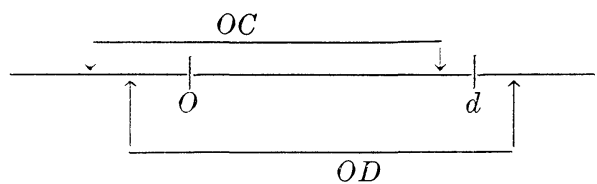

lies in the $G$-congruence class $O D,(O D) g=O D$. But $O C \cap O D$ is either a left ray of $O D$ (i.e., $s \in O C \cap O D, t \in O D$, and $t \leqq s$ implies $\mathrm{t} \in O C \cap O D$ ) or a right ray of $O D$. Since $g$ preserves order, $(O C) g$ meets $O C$ and thus $(O C) g=O C$, contradicting the assumption that $O g=d \notin O C$.

We denote the chain of convex $G$-congruences by $\Delta=\Delta(G, S)$. An ordered pair $(s, t)$ of elements of a chain $T$ is a covering pair if $s<t$ and if there is no $u \in T$ such that $s<u<t$; and we order the set of covering pairs by $(s, t)<(u, v)$ if $t \leqq u$. We denote by $\Gamma=$ $\Gamma(G, S)$ the chain of covering pairs of $\lrcorner$. We shall find in $\S 8$ that every chain occurs as $\Gamma(G, S)$ for some o-permutation group $(G, S)$. If we denote the $\gamma^{\text {th }}$ covering pair by $\gamma=\left(C_{\gamma}, C^{\gamma}\right)$, then $C^{\gamma}=C_{\eta}$ if and only if $(\gamma, \eta)$ is a covering pair of elements of $\Gamma$. Also, considering a $G$-congruence as a subset of $S \times S$, we see that $\Delta$ is closed under arbitrary intersection and union. Thus $\Delta$ is obtained from $\Gamma$ by replacing by a pair of points any point $\gamma \in \Gamma$ which is not the 
first half of a covering pair of elements of $\Gamma$, and then forming the completion by Dedekind cuts (with end points). The chains which occur as $\Delta(G, S)$ for some $(G, S)$ are those obtained in this way from an arbitrary chain $\Gamma$.

A transitive o-permutation group $(G, S)$ is said to be o-primitive if the only convex $G$-congruences are the two trivial ones. $O$-primitivity is a quite strong condition, and a great deal of information about o-primitive permutation groups is contained in [10] and [11]. Our aim in the rest of this paper is to show how an arbitrary transitive $o$-permutation group can be built up from o-primitive ones.

If $C$ and $K$ are convex $G$-congruences with $C \subset K$, it is easy to check that $K$ covers $C$ if and only if the $(C, K)$-component $\left(G_{\gamma}, S_{\gamma}\right)$ of $(G, S)$ is o-primitive. Thus the o-primitive components $\left(G_{\gamma}, S_{\gamma}\right)$ of $(G, S)$ are precisely those components arising from covering pairs $\gamma=$ $\left(C_{i}, C^{\gamma}\right)$ of convex $G$-congruences.

THEOREM 4. Let $(G, S)$ be an ordered permutation group with o-primitive components $\left(G_{\gamma}, S_{\gamma}\right)$ arising from covering pairs $\left(C_{\gamma}, C^{r}\right)$ of convex G-congruences. Then every convex G-congruence $D$ is equal to $\cap\left\{C_{r} \mid D \subseteq C_{r}\right\}$.

Proof. Since the chain $\Delta$ of all convex $G$-congruences is the Dedekind completion of the subchain consisting of those convex $G$-congruences which are (either first or second) members of covering pairs of $\Delta$, we are finished unless $C_{\gamma} \subset D \subset C^{r}$ for some $\gamma$; and this is impossible because $\left(C_{\gamma}, C^{\gamma}\right)$ is a covering pair.

An o-permutation group $\left(G_{\gamma}, S_{\gamma}\right)$ is trivial if $S_{\gamma}$ contains only one point. We now show that if the $\left(G_{\gamma}, S_{\gamma}\right)$ 's are all o-primitive and nontrivial, then $\Gamma(W, R)$ and the o-primitive components of $(W, R)$ are what they ought to be.

Theorem 5. Let $(W, R)={ }^{*} \prod_{\gamma \in \Gamma}\left(G_{\gamma}, S_{\gamma}\right)$ be the ordered wreath product of nontrivial o-primitive ordered permutation groups $\left(G_{i}, S_{\gamma}\right)$, $\Gamma$ a chain. Then the chain $\Gamma(W, R)$ of all covering pairs of convex $W$-congruences is just $\left\{\left(K_{r}, K^{r}\right) \mid \gamma \in \Gamma\right\}$, and the correspondence $\left(K_{i}, K^{r}\right) \leftrightarrow \gamma$ is an o-isomorphism between $\Gamma(W, R)$ and $\Gamma$. The $\left(K_{i}, K^{r}\right)$-component of $(W, R)$ is just $\left(G_{\gamma}, S_{i}\right)$.

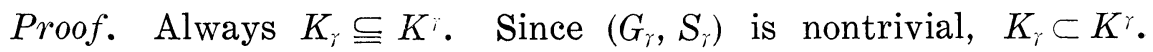
Since $\left(G_{r}, S_{\gamma}\right)$ is o-primitive, $\left(K_{\gamma}, K^{\gamma}\right)$ is a covering pair of $A(W, R)$. Now let $(C, D)$ be any covering pair of $\Delta(W, R)$. Pick $x, y \in R$ such that $(x, y) \in D \backslash C$ and let $\eta$ be the greatest element of $\{\gamma \in \Gamma \mid x(\gamma) \neq y(\gamma)\}$. Then $(x, y) \in K^{\eta} \backslash K_{\eta}$. Since $(C, D)$ and $\left(K_{\eta}, K^{\eta}\right)$ are both covering pairs, $(C, D)=\left(K_{\eta}, K^{\eta}\right)$. The rest is clear. 
COROLlaRY 6. Under the hypotheses of Theorem 5, every convex $W$-congruence $D$ is equal to $\cap\left\{K_{\gamma} \mid D \leqq K_{r}\right\}$.

5. Completeness of the ordered wreath product. The ordered wreath product $(W, R)$ has two completeness properties which we may describe as "completeness of the set $R$ " and "completeness of the group $W$ ". Although the intersection of a collection of convex congruences is always a convex congruence, it may happen in an $o$-permutation group $(G, S)$ that there is a tower of convex $G$-congruence classes (from different congruences) whose intersection is empty. An example is given in $\S 8$. The tower will be said to be complete if its intersection is not empty. If all towers are complete, then $S$ (actually $S$ together with the set of all G-congruences) is termed complete. By Theorem 4, it suffices to consider towers $\left\{x_{\lambda} C_{\lambda}: \lambda \in \Lambda\right\}$ in which every congruence is the first half of a covering pair and in which $\Lambda$ is a right ray of $\Gamma$. Henceforth we shall consider only towers of this sort. We note that if $\Gamma$ is well ordered, $S$ is automatically complete.

Theorem 7. Let $(W, R)={ }^{*} \prod_{\gamma \in I}\left(G_{\gamma}, S_{\gamma}\right)$ be the ordered wreath product of o-primitive permutation groups $\left(G_{\gamma}, S_{\gamma}\right)$. Then $R$ is complete.

Proof. Let $\left\{x_{i} K_{\lambda}: \lambda \in \Lambda\right\}$ be a tower of the prescribed kind. (We may use $K_{\gamma}$ 's because by Theorem 5 , the $\left(K_{r}, K^{\gamma}\right)$ 's constitute all covering pairs in $\Delta(W, R)$ ). Define $y$ by setting $y(\gamma)=x_{\gamma}(\gamma)$ if $\gamma \in \Lambda$; and $y(\gamma)=0(\gamma)$ otherwise. If $\lambda \in \Lambda$, then $y(\eta)=x_{\lambda}(\eta)$ for all $\eta \geqq \lambda$ because $y(\eta)=x_{\eta}(\eta)$ and $x_{\lambda} \in x_{\eta} K_{\eta}$. It follows that $y$ has inversely well ordered support, for if $\Sigma$ is contained in the support of $y$ and $\sigma \in \Sigma$, then $x_{o}(\tau)=y(\tau)$ for all $\tau \in \Sigma$ such that $\tau \geqq \sigma$, so $\Sigma$ has a greatest element. Since $y(\eta)=x_{\lambda}(\eta)$ for $\eta \geqq \lambda, y \in x_{\lambda} K_{\lambda}$ for all $\lambda \in \Delta$.

Regarding the completeness of the group, it can easily happen that an o-permutation group $(G, S)$ is a proper subgroup of an $o$ permutation group $(H, S)$ on the same set $S$, that each convex $G$-congruence is also an $H$-congruence, and yet that the $o$-primitive components of $H$ are no larger than those of $G$. (It is clear that the $\gamma$-component $\left(G_{\gamma}, S_{\gamma}\right)$ is a subgroup of the $\gamma$-component $\left.\left(H_{\gamma}, S_{\gamma}\right)\right)$. If an $o$-permutation group $(G, S)$ is not properly contained in this manner in any $(H, S)$, we shall say that the group $G$ is complete. In view of Theorem 5, the definition of ordered wreath product yields.

THEOREM 8. Let $(W, R)={ }^{*} \Pi_{\gamma \in I}\left(G_{\gamma}, S_{\gamma}\right)$ be the ordered wreath product of o-primitive permutation groups $\left(G_{\gamma}, S_{\gamma}\right)$. Then $W$ is complete. 
Let $(G, S)$ and $(H, T)$ be transitive o-permutation groups on different sets. We wish to make precise the idea of $(G, S)$ being contained in $(H, T)$ and having the same o-primitive components as $(H, T)$. Accordingly, we shall say that $(H, T)$ is a c-extension of $(G, S)$ if the following hold

(i) $S$ is a subchain of $T$.

(ii) $G$ is the faithful restriction to $S$ of some subgroup $\bar{G}$ of $H$ such that $S \bar{g}=S$ for each $\bar{g} \in \bar{G}$.

(iii) Restriction to $S$ is an isomorphism (and thus an $o$-isomorphism) from $\Gamma(H, T)$ onto $\Gamma(G, S)$. We note that (iii) holds if and only if restriction to $S$ is an isomorphism from $\Delta(H, T)$ onto $\Delta(G, S)$, which holds if and only if every convex $G$-congruence on $S$ is the restriction to $S$ of a unique convex $H$-congruence on $T$.

(iv) If $\left(D_{r}, D^{r}\right) \in \Gamma(H, T)$, and if $s \in S$, then every $D_{r}$-class contained in $s D^{r}$ meets $S$. (iv) provides a natural o-isomorphism from $S_{\gamma}$ onto $T_{\gamma}$.

(v) If $\left(D_{i}, D^{r}\right) \in \Gamma(H, T)$, and if $\mathrm{s} \in S$, then for every $h \in H$ such that $\left(s D^{r}\right) h=s D^{r}$, there exists $\bar{g} \in \bar{G}$ such that $E \bar{g}=E h$ for every $D_{r}$-class $E$ contained in $s D^{r}$. (v) provides a natural 0 -isomorphism from $G_{\gamma}$ onto $H_{\gamma}$ [8].

The concept of immediate extension in [8] specializes for ordered wreath products to the present concept of $c$-extension. It is easy to check that a $c$-extension of a $c$-extension of $(G, S)$ is itself a $c$ extension of $(G, S)$. We shall call $(G, S)$ c-closed if it has no proper $c$-extensions.

THeorem 9. Let $(G, S)$ be a transitive ordered permutation group such that $S$ and $G$ are both complete. Then $(G, S)$ is c-closed.

Proof. Suppose that $(H, T)$ is a $c$-extension of $(G, S)$. Suppose there exists $t \in T \backslash S$. Let $M$ be the intersection of all $H$-congruence classes $t B, B \in \Delta(H, T)$, such that $t B$ meets $S$. Then $M \cap S=\cap(t B \cap S)$ is not empty because $S$ is complete. Let $D$ be the convex $H$-congruence of which $M$ is a congruence class. Pick $s \in M \cap S$. Let $C$ be the union of all convex $H$-congruences $E$ such that $s E \neq t E$. Then $C$ is a convex $H$-congruence, and $D$ covers $C$. By (iv), every $C$-class contained in the $D$-class $M$ must meet $S$. In particular, $t C$ must meet $S$, contradicting the fact that $C \subset D$. Therefore $S=T$. Since $G$ is complete, $G=H$.

In the next section, we shall prove the converse of Theorem 9 .

CoRollary 10. Let $(W, R)={ }^{*} \prod_{\gamma \in \Gamma}\left(G_{\gamma}, S_{\gamma}\right)$ be the ordered wreath product of o-primitive permutation groups $\left(G_{i}, S_{\gamma}\right)$. Then $(W, R)$ is c-closed. 
6. The embedding theorem. It follows from results in [8] that if $(G, S)$ is a transitive o-permutation group with o-primitive components $\left(G_{\gamma}, S_{\gamma}\right), \gamma \in \Gamma$, then $(G, S)$ can be embedded as a permutation group in the general wreath product of the groups $\left(G_{\gamma}, S_{\gamma}\right)$. Here we show that the embedding is into the ordered wreath product and preserves order. To do so requires that we carefully describe the embedding.

Pick any $x_{0} \in S$. Our first task is to embed the chain $S$ in $R$, the set of those elements of $\prod_{r \in \Gamma} S_{r}$ with inversely well ordered support. The embedding will send $x_{0}$ onto 0 .

Lemma 11 [8]. If $H$ is any group, there exists a set $\{T(A) \mid A$ a subgroup of $H$ \} such that $T(A)$ is a set consisting of exactly one element from each right coset $A$ h of $A$ in $H, T(A) \cap A$ is the identity, and if $A \subseteq B$, then $T(A) \supseteqq T(B)$.

Now pick any $\gamma \in \Gamma$ and let $\left(G_{i}, S_{\gamma}\right)$ be the o-primitive component of $(G, S)$ determined by $\gamma=\left(C_{\gamma}, C^{\gamma}\right)$. Let $G_{x_{0}} C^{r}=\left\{g \in G \mid\left(x_{0} C^{r}\right) g=\right.$ $\left.x_{0} C^{\gamma}\right\}$. The function $\varphi: S \rightarrow \prod_{r \in I} S_{\gamma}$ is defined in the following way. Let $s \in S$. There exists exactly one $g_{s} \in T\left(G_{x_{0} C^{\gamma}}\right)$ such that $\left(x_{0} g_{s}\right) C^{r} s$. Let $(s \varphi)(\gamma)=s g_{s}^{-1} C_{\gamma} \in S_{\gamma}=\left(x_{0} C^{\gamma}\right) / C_{\gamma}$. In [8] it is shown that $\varphi$ is a one-to-one map of $S$ into $R$ and that $\left(C_{\gamma}, C^{\gamma}\right) \varphi=\left(K_{r}, K^{\gamma}\right) \mid S \varphi$, where $\left(K_{i}, K^{\gamma}\right)$ is the $\gamma^{\text {th }}$ covering pair of convex $W$-congruences (Theorem $5)$.

We show here that $\varphi$ preserves order (and in the process prove again that $\varphi$ is one-to-one). If $x<y$ are elements of $S$, let $B$ be the intersection of all convex $G$-congruences $E$ such that $x E y$, and let $A$ be the union of all convex $G$-congruences $F$ such that $x F \neq y F$. Then $(A, B)$ is a covering pair $\left(C_{r}, C^{r}\right)$ of convex $G$-congruences. Since $x C^{\gamma} y, g_{x}=g_{y}$. Thus $(x \varphi)(\gamma)=x g_{x}^{-1} C_{\gamma}<y g_{x}^{-1} C_{\gamma}=(y \varphi)(\gamma)$. However, for all $\beta>\gamma, C^{r} \subseteq C_{\beta}$, so that $x C_{\beta} y$, and hence $(x \varphi)(\beta)=(y \varphi)(\beta)$. Therefore $x \varphi<y \varphi$. Thus the chain $S$ is $o$-embedded in the chain $R$.

Next we define an embedding (also denoted by $\varphi$ ) of $G$ in $W$. Let $g \in G, \gamma \in \Gamma$, and $x \in R$. $g$ induces an $o$-permutation of $S_{r}$, which is formally denoted by $(g \varphi)_{i, x}$, as follows. If $x K^{\gamma}$ meets $S \varphi$, then for each $a \in S_{\gamma}$, there exists $s \in S$ such that $s \varphi \in x K^{\gamma}$ and $(s \varphi)(\gamma)=a$. Now define $a(g \varphi)_{\gamma, x}=((s g) \varphi)(\gamma)$. If $x K^{\gamma}$ does not meet $S \varphi$, define $(g \varphi)_{i, x}$ to be the identity $1_{\gamma}$ on $S_{\gamma}$. In [8] it is shown that $(g \varphi)_{r, x}$ is well defined and is a permutation of $S_{\gamma}$. Here we show it preserves order. Suppose we have $a, b \in S_{r}$ with $a<b$, and $s, t \in S$ with $(s \varphi) K^{\gamma} x K^{\gamma}(t \varphi)$ and $(s \varphi)(\gamma)=a,(t \varphi)(\gamma)=b$. Then $s \varphi<t \varphi$, so $s<t$, so $s g<t g$, so $(s g) \varphi<(t g) \varphi$. Since $(s \varphi) K^{\gamma}(t \varphi)$, we have $s C^{\gamma} t$, so $(s g) C^{\gamma}(t g)$, and thus $((s g) \varphi) K^{\gamma}((t g) \varphi)$. Since also $((s g) \varphi) K_{\gamma} \neq((t g) \varphi) K_{\gamma}$, we have $a(g \varphi)_{\gamma, x}=((s g) \varphi)(\gamma)<((t g) \varphi)(\gamma)=b(g \varphi)_{\gamma, x}$. Thus $(g \varphi)_{\gamma, x}$ pre- 
serves order.

Now $g \varphi$ is defined as a function on $R$ by setting $(r(g \varphi))(\gamma)=$ $(r(\gamma))(g \varphi)_{\gamma, r}$. It is proved in [8] that $g \varphi$ is a permutation of $R$, and moreover is contained in the general wreath product; and that its component $(g \varphi)_{r, x}$ defined in $\S 3$ coincides with the permutation which is formally denoted above by $(g \varphi)_{r, x}$. By Theorem 1, $g \varphi$ preserves order on $R$. It was also shown in [8] that $\varphi$ is an embedding of the group $G$ in $W$ satisfying $(s g) \varphi=(s \varphi)(g \varphi)$ for all $s \in S, g \in G$. Thus $g$ is a positive $o$-permutation of $S$ if and only if $g \varphi$ is a positive $o$-permutation of $S \varphi$.

To show that $\varphi: G \rightarrow W$ is an o-embedding, it remains to show that if $g \varphi$ is positive on $S \varphi$, it is also positive on $R$. Thus suppose that $g \varphi$ is positive on $S \varphi$ and let $\mathrm{r} \in R$. Let $\gamma$ be the largest element of $\Gamma$ such that $r(\gamma) \neq(r(g \varphi))(\gamma)$. We may assume that $r K^{\gamma}$ meets $S \varphi$ (otherwise $\left.(r(g \varphi))(\gamma)=r(\gamma)(g \varphi)_{\gamma, r}=r(\gamma) 1_{\gamma}=r(\gamma)\right)$ and thus that there exists $s \varphi \in r K_{\gamma}$. Since $g \varphi$ is positive on $S \varphi$ and since $\left((s \varphi) K^{r}\right)(g \varphi)=$ $(s \varphi) K^{\gamma}$, we have $r(\gamma)=(s \varphi)(\gamma)<((s \varphi)(g \varphi))(\gamma)=(r(g \varphi))(\gamma)$. Therefore $g \varphi$ is positive on $R$, and thus $\varphi: G \rightarrow W$ is an $o$-embedding. It follows easily (and is essentially included in [8]) that $(W, R)$ is a $c$-extension of $(G \varphi, S \varphi)$. We recapitulate the foregoing discussion in the following theorem.

THEOREM 12. Let $(G, S)$ be a transitive ordered permutation group with o-primitive components $\left(G_{\gamma}, S_{\gamma}\right), \gamma \in \Gamma$. Let $(W, R)$ be the ordered wreath product ${ }^{*} \Pi_{\gamma \in \Gamma}\left(G_{\gamma}, S_{\gamma}\right)$. Then there is an embedding $\varphi:(G, S) \rightarrow(W, R)$ of the following sort. First, $\varphi: S \rightarrow R$ is an 0 embedding, and $\left(C_{r}, C^{\gamma}\right) \varphi=\left(K_{r}, K^{\gamma}\right) \mid S \varphi . \quad$ Next, $\varphi: G \rightarrow W$ is an oembedding of the partially ordered group $G$ in the partially ordered group W. Finally, $(s \varphi)(g \varphi)=(s g) \varphi$ for all $s \in S, g \in C$; and $(W, R)$ is $a$ (c-closed) c-extension of $(G \varphi, S \varphi)$.

CoRollary 13. Let $(G, S)$ be a transitive ordered permutation group. Then the following are equivalent:

(1) $(G, S)$ is o-isomorphic to the ordered wreath product $(W, R)$ of its o-primitive components.

(2) $(G, S)$ is c-closed.

(3) $S$ and $G$ are both complete.

Proof. (1) implies (3) by Theorems 7 and 8. (3) implies (2) by Theorem 9. (2) implies (1) by Theorem 12.

CoROLlaRY 14. Let $(G, S)$ be a transitive ordered permutation group and $(W, R)={ }^{*} \prod_{r \in \Gamma}\left(G_{\gamma}, S_{\gamma}\right)$ the ordered wreath product of its 
o-primitive components. Then any other c-closed c-extension $(H, T)$ of $(G, S)$ is o-isomorphic to $(W, R)$.

Proof. Since $(H, T)$ is a $c$-extension of $(G, S)$, the ordered wreath product of its o-primitive components is also $(W, R)$. Since $(H, T)$ is $c$-closed, $(H, T)$ is o-isomorphic to $(W, R)$.

We now identify $(G, S)$ with $(G \varphi, S \varphi)$ and describe the way in which $S$ has been filled out to obtain $R$. Let $\left\{x_{\lambda} C_{\lambda} \mid \lambda \in \Lambda\right\}$ be an incomplete tower of convex $G$-congruence classes in $S$. By Theorem 7, $M=\cap\left\{x_{\lambda} K_{\lambda} \mid \lambda \in A\right\} \subseteq R \backslash S$ is not empty. Thus $M$ is a convex congruence class of the $W$-congruence $\cap\left\{K_{\lambda} \mid \lambda \in \Lambda\right\}$; and consists of more than one point if and only if $A \neq \Gamma . \quad M$ has been inserted in the Dedekind cut of $S$ which corresponds to the left ray $\{s \in S \mid$ there exists $\lambda=\lambda_{s}$ with $s<y$ for all $\left.y \in x_{\lambda} C_{\lambda}\right\}$. Conversely, every $r \in R \backslash S$ arises in this way from the incomplete tower $\left\{x_{\lambda} C_{\lambda} \mid \lambda \in \Lambda\right\}$ defined by $x_{\lambda} \in r K_{\lambda} \cap S$ if $r K_{\lambda}$ meets $S$; and $x_{\lambda}$ undefined otherwise. Clearly $x_{\lambda} C_{\lambda}$. depends only on $\lambda$; and $\cap\left\{x_{\lambda} C_{\lambda} \mid \lambda \in \Lambda\right\}$ is empty, for if $s \in \cap\left\{x_{\lambda} C_{\lambda}\right\}$, then $r K_{r}$ meets $S$ for some $\gamma \notin \Lambda$.

In particular, $S=R$ if and only if $S$ is complete (as we had essentially shown already); and $S$ is order dense in $R$ if and only if for every incomplete tower $\left\{x_{\lambda} C_{2}: \lambda \in \Lambda\right\}, \Lambda=\Gamma$. Also, it is not hard to show that for $x \in S, x K^{\gamma}$ is the convex hull of $x C^{\gamma}$, i.e., for any $y \in x K^{\gamma}$, there exist $u, v \in x C^{\gamma}$ such that $u \leqq y \leqq v$.

7. Lattice-ordered permutation groups. If $\left(G_{\gamma}, S_{\gamma}\right)$ is an ordered permutation group, so that $G_{\gamma}$ is a subgroup of $A\left(S_{\gamma}\right)$, then if $G_{r}$ is closed under the lattice operations, $G_{\gamma}$ is a lattice-ordered group ( $l$ group). In this case we shall call $\left(G_{\gamma}, S_{\gamma}\right)$ a lattice-ordered permutation group.

THEOREM 15. If $(G, S)$ is a transitive lattice-ordered permutation group and $\gamma=\left(Q_{r}, Q^{r}\right)$ is a pair of convex G-congruences with $Q_{r} \subset Q^{r}$, then the component $\left(G_{\gamma}, S_{\gamma}\right)$ is also a lattice-ordered permutation group.

Proof. $G_{O Q^{r}}$ is an $l$-subgroup of $G$; and the map $g \rightarrow \bar{g}$ from $G_{O Q^{r}}$ into $A\left(S_{\gamma}\right)$ is an $l$-homomorphism since

$$
\left(x Q_{\gamma}\right)(\overline{g \vee 1})=(x(g \vee 1)) Q_{\gamma}=(x g \vee x) Q_{\gamma}=(x g) Q_{\gamma} \vee x Q_{\gamma}=\left(x Q_{\gamma}\right)(\bar{g} \vee \overline{1}) .
$$

Thus the image $G_{\gamma}$ is an $l$-subgroup of $A\left(S_{\gamma}\right)$.

Theorem 16 [10]. If each $\left(G_{\gamma}, S_{\gamma}\right)$ is a transitive lattice-ordered permutation group, then the ordered wreath product $(W, R)=$ ${ }^{*} \Pi_{\gamma \in \Gamma}\left(G_{r}, S_{\gamma}\right)$ is also a (transitive) lattice-ordered permutation group, and conversely. 
Proof. The converse follows from the previous theorem. Suppose now that each $\left(G_{\gamma}, S_{\gamma}\right)$ is lattice-ordered. Let $w \in W$ have as components $\left\{w_{\gamma, x}\right\}$. Define $z$ by setting $z_{r, x}$ equal to

(i) $w_{r, x}$ if $(x w) K^{r}>x K^{r}$,

(ii) $1_{r}$ if $(x w) K^{r}<x K^{r}$,

(iii) $w_{r, x} \vee 1_{r}$ if $(x w) K^{r}=x K^{r}$ (i.e., if $\left.(x w) K^{r} x\right)$. $z_{r, x}$ depends only on $x K^{\gamma}$, for if also $y \in x K^{\gamma}$, then $x K^{\gamma} y$, so $(x w) K^{\gamma}(y w)$, and thus the same one of the three cases applies. If $\delta$ is the greatest element of $\Gamma$ at which $s w$ differs from $s$, then $s z=s w$ if $(s w)(\delta)>s(\delta)$, while $s z=s$ if $(s w)(\delta)<s(\delta)$, so that $z=w \vee 1$.

THEOREM 17. Let $(G, S)$ be a transitive lattice-ordered permutation group. Then the embedding $\varphi: G \rightarrow W$ described in Theorem 12 is an l-isomorphism.

Proof. Since $\varphi: G \rightarrow G \varphi$ is an $o$-isomorphism, it is an $l$-isomorphism, and thus for all $s \in S,(s \varphi)((g \vee 1) \varphi)=(s \varphi)(g \varphi) \vee s \varphi$. Now let $r \in R$, $\gamma \in \Gamma$. If there exists $s \in S$ such that $(s \varphi) K_{\gamma} r$, then

$$
\begin{aligned}
{[r((g \vee 1) \varphi)](\gamma) } & =[s \varphi((g \vee 1) \varphi)](\gamma)=[(s \varphi)(g \varphi) \vee s \varphi](\gamma) \\
& =[r(g \varphi) \vee r](\gamma)=[r(g \varphi \vee 1)](\gamma) .
\end{aligned}
$$

If, on the other hand, there is no such $s$, then $[r((g \vee 1) \varphi)](\gamma)=$ $r(\gamma)=[r(g \varphi \vee 1)](\gamma)$. Therfore $r((g \vee 1) \varphi)=r(g \varphi \vee 1)$, so that $\varphi$ is an $l$-isomorphism.

If $(G, S)=(A(S), S)$, the situation is even nicer. The 0 -primitive component $\left(G_{\gamma}, S_{\gamma}\right)$ is $\left(A\left(S_{\gamma}\right), S_{\gamma}\right)$. It is shown in [9] that if $(A(T), T)$ is 0 -primitive, then it is either 0 -2-transitive (i.e., for $t_{1}<t_{2}$ and $u_{1}<u_{2}$, there exists $g \in A(T)$ such that $t_{1} g=u_{1}$ and $\left.t_{2} g=u_{2}\right)$ or the regular representation of a rather special subgroup of the reals. Also, if $S$ is complete, the embedding of Theorem 12 maps $A(S)$ onto $W$ (as well as mapping $S$ onto $R$ ).

8. Totally ordered groups. If $G$ is a totally ordered group, then the right regular representation $(G, G)$ is an o-permutation group. A convex $G$-congruence amounts to a partition of $G$ by the right cosets $L g$ of some convex subgroup $L$ of $G$; and the ordering of the convex $G$-congruences corresponds to the ordering by inclusion of the convex subgroups of $G$.

Thus an o-primitive component of $(G, G)$ arises from a covering pair $\left(C_{r}, C^{r}\right)$ of convex subgroups of $G$. Hence in the o-primitive component $\left(G_{\gamma} S_{\gamma}\right), S_{\gamma}$ is just the chain $C^{r} / C_{r}$. It is well kown [3] that $C_{r}$ is normal in $C^{r}$ and that the quotient group $C^{r} / C_{r}$ is o-isomorphic to a subgroup of the real numbers. The kernel of the natural 
map $g \rightarrow \bar{g}$ from the group $C^{r}$ into $A\left(S_{\gamma}\right)$ is $\left\{g \in C^{\gamma} \mid\left(C_{\gamma} x\right) g=C_{\gamma} x\right.$ for all $x \in C^{\gamma}$, which is just $C_{\gamma}$ since $C_{\gamma}$ is normal in $C^{\gamma}$. Hence $G_{\gamma}$ is oisomorphic to the o-group $C^{r} / C_{r}$, a subgroup of the reals. Thus $\left(G_{r}, S_{r}\right)$ is just the regular representation of a subgroup of the reals. Using only the group embedding $\varphi: G \rightarrow W$ of Theorem 12, we have an analogue of Hahn's theorem.

THEOREM 18. If $G$ is a totally ordered group, then $G$ can be oembedded in an ordered wreath product of subgroups of the real numbers.

As mentioned before, this theorem is somewhat unsatisfactory since the wreath product is not totally ordered, but by Theorem 16 , it is at least lattice-ordered.

We mention without proof that if the transversals of Lemma 11 can be chosen so that for each $\gamma \in \Gamma, G$ is the direct sum of $G_{x_{0} C^{r}}$ and $T\left(C_{x_{0} c} \gamma\right.$, then the embedding $\varphi$ can be chosen so that for each $g \in G, \gamma \in \Gamma,(g \varphi)_{\gamma, x}=(g \varphi)_{\gamma, y}$ for all $x, y \in S$, i.e., all of the $\gamma$-components of $g$ are the same. In this case, $\varphi$ embeds $G$ in the (unrestricted) direct product $\prod_{r \in \Gamma} G_{\gamma}$. According to the lemma of Banaschewski [1], this hypothesis is satisfied if $G$ is a divisible totally ordered abelian group. Thus Hahn's theorem is a corollary of the theory developed here. However, a proof of Hahn's theorem along these lines is essentially the same as the proof in [1].

Hahn groups provide several examples which help to clarify the theory of ordered wreath products. Let $\Gamma$ be an arbitrary chain and let $G$ be a Hahn group on $\Gamma$. Then $\Gamma(G, G)=\Gamma$, so every chain $\Gamma$ occurs as $\Gamma(G, S)$ for some o-permutation group $(G, S)$. Let $H$ be a restricted Hahn group on $\Gamma$ (containing only elements having finite support). If $\Gamma$ is the chain $N$ of negative integers, $(H, H)$ has incomplete towers, but at least every incomplete tower has $\Lambda=\Gamma$. If $\Gamma$ is the ordinal sum $N+N,(H, H)$ has imcomplete towers with $\Lambda \neq \Gamma$.

\section{REFERENCES}

1. B. Banaschewski, Totalgeordnete Moduln, Archiv. Math. 7 (1956), 430-440.

2. P. Conrad, Non-abelian ordered groups, Pacific J. Math. 9 (1959), 25-41.

3. L. Fuchs, Partially ordered algebraic systems, Addison-Wesley, Reading, Mass., 1963.

4. H. Hahn, Über die nichtarchimedische Grössensysteme, S.-B. Kaiserlichen Akad. Wiss. Math. Nat. Kl. IIa, 116 (1907), 601-655.

5. M. Hall, The theory of groups, MacMillan, New York, 1959.

6. W. C. Holland, The lattice-ordered group of automorphisms of an ordered set, Michigan Math. J. 10 (1963), 399-408.

7. - Transitive lattice-ordered permutation groups, Math. Zeit. 87 (1965), 
420-433.

8. - The characterization of generalized wreath products (to appear in J. of Algebra).

9. J. T. Lloyd, Lattice-ordered groups and o-permutation groups, Dissertation, Tulane University, 1964.

10. S. H. McCleary, Orbit configurations of ordered permutation groups, Dissertation, University of Wisconsin, 1967.

11. - Orbits of stabilizer subgroups of ordered permutation groups (to appear)

12. N. Reilly, Some applications of wreath products and ultra-products in latticeordered groups (to appear).

Received May 6, 1969. The research for this paper was supported in part by the National Science Foundation.

UNIVERSITY OF WISCONSIN

UNIVERSITY OF GEORGIA 


\title{
PACIFIC JOURNAL OF MATHEMATICS
}

\author{
EDITORS
}

\author{
H. ROYDEN \\ Stanford University \\ Stanford, California \\ RICHARD PIERCE \\ University of Washington \\ Seattle, Washington 98105
}

\author{
J. DUGUNDJI \\ Department of Mathematics \\ University of Southern California \\ Los Angeles, California 90007 \\ BASIL GORDON \\ University of California \\ Los Angeles, California 90024
}

\section{ASSOCIATE EDITORS}

E. F. BECKENBACH
B. H. NeumanN

F. WOLF
K. YoshidA

\section{SUPPORTING INSTITUTIONS}

\author{
UNIVERSITY OF BRITISH COLUMBIA \\ CALIFORNIA INSTITUTE OF TECHNOLOGY \\ UNIVERSITY OF CALIFORNIA \\ MONTANA STATE UNIVERSITY \\ UNIVERSITY OF NEVADA \\ NEW MEXICO STATE UNIVERSITY \\ OREGON STATE UNIVERSITY \\ UNIVERSITY OF OREGON \\ OSAKA UNIVERSITY \\ UNIVERSITY OF SOUTHERN CALIFORNIA
}

\author{
STANFORD UNIVERSITY \\ UNIVERSITY OF TOKYO \\ UNIVERSITY OF UTAH \\ WASHINGTON STATE UNIVERSITY \\ UNIVERSITY OF WASHINGTON

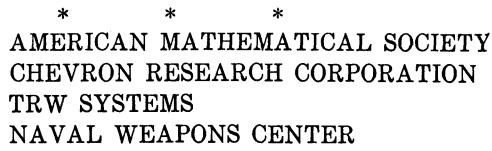

The Supporting Institutions listed above contribute to the cost of publication of this Journal, but they are not owners or publishers and have no responsibility for its content or policies.

Mathematical papers intended for publication in the Pacific Journal of Mathematics should be in typed form or offset-reproduced, double spaced with large margins. Underline Greek letters in red, German in green, and script in blue. The first paragraph or two must be capable of being used separately as a synopsis of the entire paper. It should not contain references to the bibliography. Manuscripts, in duplicate if possible, may be sent to any one of the four editors. Please classify according to the scheme of Math. Rev. 36, 1539-1546. All other communications to the editors should be addressed to the managing editor, Richard Arens, University of California, Los Angeles, California, 90024.

50 reprints are provided free for each article; additional copies may be obtained at cost in multiples of 50 .

The Pacific Journal of Mathematics is published monthly. Effective with Volume 16 the price per volume (3 numbers) is $\$ 8.00$; single issues, $\$ 3.00$. Special price for current issues to individual faculty members of supporting institutions and to individual members of the American Mathematical Society: $\$ 4.00$ per volume; single issues $\$ 1.50$. Back numbers are available.

Subscriptions, orders for back numbers, and changes of address should be sent to Pacific Journal of Mathematics, 103 Highland Boulevard, Berkeley, California, 94708.

PUBLISHED BY PACIFIC JOURNAL OF MATHEMATICS, A NON-PROFIT CORPORATION

Printed at Kokusai Bunken Insatsusha (International Academic Printing Co., Ltd.), 7-17, Fujimi 2-chome, Chiyoda-ku, Tokyo, Japan. 


\section{Pacific Journal of Mathematics}

\section{Vol. 31, No. $3 \quad$ BadMonth, 1969}

George E. Andrews, On a calculus of partition functions .................. 555

Silvio Aurora, A representation theorem for certain connected rings ............ 563

Lawrence Wasson Baggett, A note on groups with finite dual spaces ............. 569

Steven Barry Bank, On majorants for solutions of algebraic differential equations in regions of the complex plane ............................... 573

Klaus R. Bichteler, Locally compact topologies on a group and the corresponding continuous irreducible representations ......................... 583

Mario Borelli, Affine complements of divisors ....................... 595

Carlos Jorge Do Rego Borges, A study of absolute extensor spaces ............. 609

Bruce Langworthy Chalmers, Subspace kernels and minimum problems in Hilbert

spaces with kernel function ...................................... 619

John Dauns, Representation of L-groups and F-rings................. 629

Spencer Ernest Dickson and Kent Ralph Fuller, Algebras for which every

indecomposable right module is invariant in its injective envelope ...........

Robert Fraser and Sam Bernard Nadler, Jr., Sequences of contractive maps and fixed

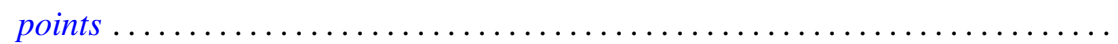

Judith Lee Gersting, A rate of growth criterion for universality of regressive

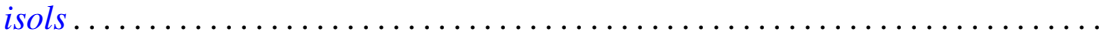

Robert Fred Gordon, Rings in which minimal left ideals are projective ............

Fred Gross, Entire functions of several variables with algebraic derivatives at certain algebraic points

W. Charles (Wilbur) Holland Jr. and Stephen H. McCleary, Wreath products of ordered permutation groups .........................

W. J. Kim, The Schwarzian derivative and multivalence .................. 717

Robert Hamor La Grange, Jr., On $(\mathrm{m}-\mathrm{n})$ products of Boolean algebras ......... 725

Charles D. Masiello, The average of a gauge ........................ 733

Stephen H. McCleary, The closed prime subgroups of certain ordered permutation

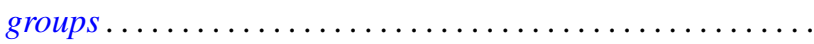

Richard Roy Miller, Gleason parts and Choquet boundary points in convolution measure algebras ...............................

Harold L. Peterson, Jr., On dyadic subspaces ........................ 773

Derek J. S. Robinson, Groups which are minimal with respect to normality being intransitive........................................... 777

Ralph Edwin Showalter, Partial differential equations of Sobolev-Galpern type . . . 787

David Slepian, The content of some extreme simplexes ................... 795

Joseph L. Taylor, Noncommutative convolution measure algebras ............. 809

B. S. Yadav, Contractions of functions and their Fourier series ............... 827

Lindsay Nathan Childs and Frank Rimi DeMeyer, Correction to automorphisms of separable algebras" ....................... 833

Moses Glasner and Richard Emanuel Katz, Correction to: "Function-theoretic degeneracy criteria for Riemannian manifolds".............

Satish Shirali, Correction to: "On the Jordan structure of complex Banach

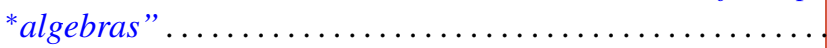

